\title{
Severe erythema multiforme-type drug eruption controlled by tumor necrosis factor- $\alpha$ antagonist: A case study
}

\author{
XIN LING ${ }^{1}, \mathrm{XIN} \mathrm{SHI}^{2}$ and LINGLING CHEN ${ }^{3}$ \\ ${ }^{1}$ Department of Dermatology, The First People's Hospital of Wujiang, Affiliated Wujiang Hospital of Nantong University, \\ Suzhou, Jiangsu 215200; ${ }^{2}$ Department of Dermatology, The Second Affiliated Hospital of Soochow University, Suzhou, \\ Jiangsu 215004; ${ }^{3}$ Department of Dermatology, Suzhou Municipal Hospital, Suzhou, Jiangsu 215006, P.R. China
}

Received May 9, 2016; Accepted March 31, 2017

DOI: $10.3892 /$ etm.2017.5336

\begin{abstract}
Using a tumor necrosis factor- $\alpha$ antagonist, the present study successfully treated a case of severe erythema multiform-type drug eruption, which occurred following anti-Helicobacter pylori therapy. A 73-year-old female suffering from upper gastrointestinal bleeding and peptic-ulcer presented with an itchy rash, fever, an increase in leukocytes and eosinophils and lymphadenectasis following oral administration of amoxicillin. Following six subcutaneous injections of etanercept (initially $50 \mathrm{mg}$, then $25 \mathrm{mg}$ every 3 days), the patient was deemed to have recovered. Following the first injection, the fever was under control. On day 2, the lesions were no longer expanding. On day 4 , the rash was markedly less itchy, the swelling decreased, the erythema began to crust and mucosal secretions disappeared. On day 16, the patient was deemed to have recovered and was discharged from the hospital. Her peripheral blood eosinophil count continued to rise following the injection, peaking on day 9. Following this, the count declined slowly, but remained significantly higher than normal when the patient was discharged. The present case indicates that tumor necrosis factor- $\alpha$ antagonist is a safe, fast and effective treatment for severe drug eruption, but it is unable to prevent the rise of peripheral blood eosinophils.
\end{abstract}

\section{Introduction}

Clinically, severe drug eruption is typically divided into toxic epidermal necrolysis (TEN), severe erythema multiforme-type drug eruption (Steven-Johnson syndrome; SJS) and exfoliative dermatitis (Erythrodermic drug eruption). Severe drug eruption is a serious disease that is difficult to treat and has a high mortality rate (1); for example, the mortality rate of TEN is

Correspondence to: Dr Lingling Chen, Department of Dermatology, Suzhou Municipal Hospital, 26 Daoqian Street, Suzhou, Jiangsu 215006, P.R. China

E-mail: chenlingling031@126.com

Key words: drug eruption, tumor necrosis factor- $\alpha$ antagonist, therapeutic outcome
$30 \%$ (2). Corticosteroids are the preferred therapeutic for severe drug eruption, however, the use of corticosteroids to treat severe drug eruption is clinically controversial (2). Engelhardt et al (3) hypothesized that the use of corticosteroids would not be able to shorten the course of drug eruption. Instead, it would increase the risk of infection, sepsis and gastrointestinal bleeding, prolonging hospital stays and increasing mortality. A study by Guibal et al (4) demonstrated that although long-term corticosteroid therapy may delay the progression of TEN, it was not able to prevent the entire progression of the disease. Schneck et al (5) revealed that the use of corticosteroids was not beneficial in the reduction of SJS and TEN-associated mortality in a large-scale retrospective study in France and Germany.

Due to the side effect of corticosteroids, the use of corticosteroids is restricted for patients with underlying diseases, including diabetes, gastrointestinal bleeding, peptic-ulcer, epilepsy, glaucoma, cataracts, osteoporosis, tuberculosis or hypertension (6). In the present study, the patient was admitted to hospital due to upper gastrointestinal bleeding. Following anti-Helicobacter pylori (HP) treatment, the patient presented with severe drug eruption. Routine use of corticosteroids may have, in the present case, increased the risk of aggravating the underlying disease. Previous studies in which tumor necrosis factor (TNF)- $\alpha$ antagonists have been successfully used to treat drug eruption have been reported $(2,7,8)$.

A TNF- $\alpha$ antagonist was subsequently used as treatment and the patient was fully recovered after six injections. Rash, pyrexia, peripheral blood leukocytes, eosinophils and the evolution of the systematic damage were assessed and reported.

\section{Case study}

Patient. In May 2015, a 73-year-old female was admitted to the gastroenterology clinic of The First People's Hospital of Wujiang (Suzhou, China) due to abdominal pain that had persisted for 1 week and melena for 5 days. Endoscopy indicated the presence of a compound gastric ulcer with bleeding. Pathological diagnosis demonstrated that the antral gastric mucosa exhibited chronic active inflammation. Following treatment with amoxicillin, clarithromycin, pantoprazole, and colloidal bismuth pectin (quadruple anti-HP therapy), the patient's abdominal pain eased. On day 4 , an itchy erythema 
appeared around the patient's neck and the patient was re-admitted to hospital.

The patient presented with no cough or expectoration in the course of treatment. The patient had a history of hypertension and was taking antihypertensive tablets daily to control her blood pressure. No history of coronary heart disease, trauma, blood transfusion or drug allergy was indicated. The patient's stool was black and urine frequency, output and color were normal.

Examination. Clinical examination demonstrated that the patient's temperature was $37.4^{\circ} \mathrm{C}$ (Fig. 1), pulse was 86 beats per minute, blood pressure was $144 / 79 \mathrm{mmHg}$, respiratory rate was 18 beats per minute and $\mathrm{SpO}_{2}$ was $96 \%$. The skin and mucous membranes across the entire body were not cyanosed, icteric and pale. Superficial lymph nodes were impalpable. Lung breath sounds were rough but rhonchi and moist rale were not heard and the heartbeat was regular. Pathological cardiac murmurs were not heard in the auscultation area of the heart valves. The abdomen was soft, with upper abdominal tenderness below the xiphoid process. Abdomen had no rebound tenderness or muscle tension and abdominal mass was impalpable. The liver and spleen were impalpable below the costal margin. Murphy's sign, shifting dullness and renal percussive pain were all negative. Bowel sounds occurred 5 times per min. Lower limbs had no edema. Physiological reflex was normal and pathologic reflex was not elicited.

Skin changes. Edematous erythema appeared on the head, face and neck. The eyelids slightly swelled but the conjunctiva presented with no congestion. The mouth and genitalia had no ulceration or erosion.

Laboratory examination. Laboratory examinations were conducted and the findings were as follows: Leukocyte count, $5.03 \times 10^{9}$ cells/1 (normal, 4.00-10.0× $10^{9}$ cells/l); hemoglobin (Hb), $105 \mathrm{~g} / 1$ (normal, 110-150 g/1); neutrophil ratio, 74.7\% (normal, 50-70\%); lymphocyte ratio, 14.3\% (normal, 20 40\%); eosinophil ratio, $5.2 \%$ (normal, $0.5-5.0 \%$ ); erythrocyte sedimentation rate, $19 \mathrm{~mm} / \mathrm{h}$ (normal, 0-20 mm/h); glycated hemoglobin, 5.4\% (normal, 4.0-6.0\%); total glycated $\mathrm{Hb}$, $6.4 \%$ (normal, 3.9-7.3\%); C-reactive protein, $2 \mathrm{mg} / 1$ (normal, 0.00-10.00 mg/l); ketone, 2+ (normal, 0-0.5 mmol/1); urine protein, negative; D-dimer, $1.23 \mathrm{mg} / \mathrm{l}$ (normal, 0.00-0.55 mg/l) and total T3 $0.92 \mathrm{nmol} / 1$ (normal, 1.34 2.73 nmol/l).

Gastroscopy (pre-admission). The patient presented with a compound gastric ulcer with bleeding. Pathological diagnosis demonstrated that the antral gastric mucosa presented with chronic active inflammation. Preliminary diagnosis was a compound gastric ulcer with bleeding and drug eruption (slight) following treatment.

Admission course and treatment procedure. The patient's rash further developed following admittance to hospital. The rash on the face, neck, trunk and limbs had worsened and the patient also had itchiness, fever and increased peripheral blood leukocytes. Following intravenous injection of $60 \mathrm{ml}$ compound glycyrrhizin $(20 \mathrm{ml}$ contains $40 \mathrm{mg}$ glycyrrhizin, $400 \mathrm{mg}$ aminoacetic acid and $20 \mathrm{mg}$ cysteine hydrochloride;
Minophagen Pharmaceutical Co,. Ltd., Tokyo, Japan) and administration of calamine lotion, the rash further increased and integrated. The face became swollen, the conjunctiva exhibited hyperemia and edema and the conjunctival secretion increased. On day 3 following admission, the patient presented with a fever at 6:00 a.m. (body temperature, $38.7^{\circ} \mathrm{C}$; Fig. 1). The rash was more swollen and reddish, and had integrated further. Conjunctival secretion markedly increased and the patient had difficulty opening their eyes. Typical target-like erythematous papules appeared on the lower limbs. On day 4, the patient's condition aggravated further. The rash extended to cover $80 \%$ of the body surface area and the skin temperature increased (Fig. 2A-C) with the patient indicating that the itch was severe. Her body temperature rose to $39.6^{\circ} \mathrm{C}$ (Fig. 1) at 6:00 p.m. Leukocyte and neutrophil counts markedly increased as determined by laboratory examination: Leukocyte count, 24.70 $\times 10^{9} / 1$; neutrophil ratio, 93\%; and eosinophil ratio, $1.9 \%$. A diagnosis of severe drug eruption (severe erythema multiforme-type drug eruption) was given.

The patient was hospitalized due to upper gastrointestinal bleeding and a peptic ulcer. Following anti-HP treatment, a severe drug eruption occurred. Therefore, the routine use of corticosteroids posed an increased risk to this patient. The patient was assessed for viral hepatitis, cancer and tuberculosis through hepatitis series, tumor series and X-ray testing, all of which were negative. Written informed consent was provided by the patient, prior to the subcutaneous injection of the recombinant human TNF receptor II-antibody fusion protein (etanercept) with a $25 \mathrm{mg} /$ needle (CITIC National Health Pharmaceutical Co., Ltd., Shanghai, China) every three days with the initial dose being $50 \mathrm{mg} /$ day and the maintenance dose as $25 \mathrm{mg}$ /day. Following six injections with the protein, the patient's symptoms were improved.

As severe drug eruption lacks acknowledged evaluation criteria, the present study referred to an eruption-severity scoring method, outlined by Chen et al (8), that utilizes a drug eruption area and severity index (DASI). According to this, the evolution of the rash was recorded and quantified. The method was as follows: i) Evaluating rash area (A). Head/neck made up $10 \%$, trunk made up $30 \%$, upper limbs made up $20 \%$, and lower limbs made up $40 \%$. If no rash was observed, the area was assigned a score of 0 . The present study assigned points as follows: 1 , rash area $<10 \% ; 2,10-29 \% ; 3,30-49 \%$; $4,50-69 \% ; 5,70-89 \%$; and 6, 90-100\%. Overall rating scores were between 0 and 24 points. Upper limbs included axillas and hands. The trunk included the groin and middle axillary axial area. Lower limbs included hips and feet. ii) Evaluating the rash severity. The following features were evaluated: Erythema (E), infiltration or edema (I), erosion or vesicle (Ev), and desquamation (D). Each feature was evaluated using a 0-3 rating: 0 , none; 1 , slight condition; 2 , moderate condition; and 3 , severe condition. D was scored in reverse. iii) Evaluating mucosal damage. The mucosa included the eyes, nose, mouth, genitalia, anus and rectum. Involvement of the eyes and oral mucosa scored 2 points and the rest scored 1 point. Severe eye mucosal damage may cause blindness and severe oral mucosal damage affects eating and therefore, the involvement of two mucosal areas is very important. Severity of mucosal damage was scored using a 4-point system, as follows: 0 point, none; 1 point, $<5$ blisters; 2 points, $>5$ blisters and (or) mild erosion; 


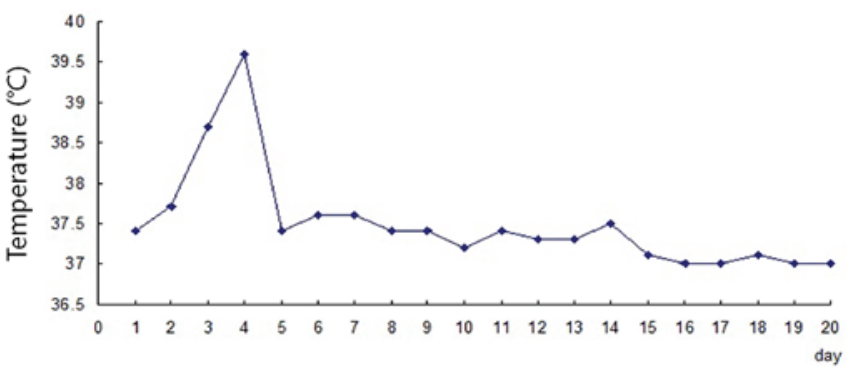

Figure 1. Patient's daily maximum temperature changes following hospital admittance.
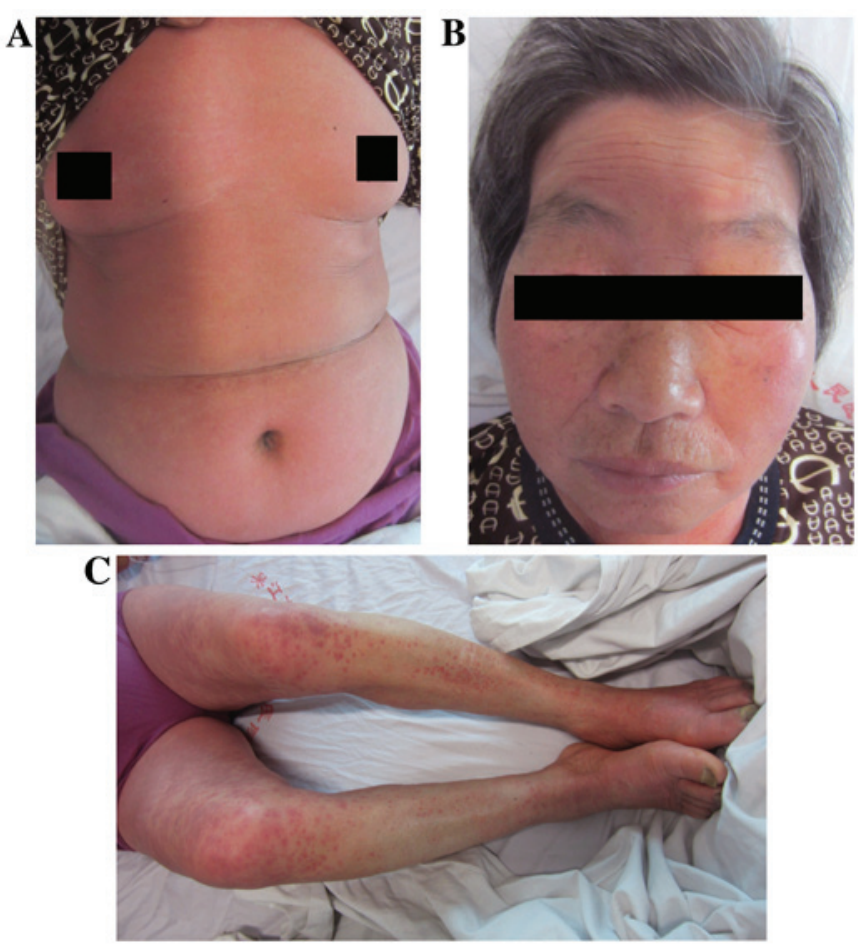

Figure 2. On the first day of the initial dose, the patient presented with generalized edematous erythema, conjunctival erosion, significantly increased secretion, she had difficulty opening her eyes and exhibited a high fever and severe itch. The patient's (A) torso, (B) face and (C) legs are presented to demonstrate the extent of the rash.

and 3 points, severe erosion. DASI total scores were calculated as follows: DASI total scores $=($ E head $+\mathrm{I}$ head + Ev head + D head) x A head x $0.1+($ E upper limbs + I upper limbs + Ev upper limbs + D upper limbs) x A upper limbs x $0.2+(\mathrm{E}$ trunk $+\mathrm{I}$ trunk $+\mathrm{Ev}$ trunk $+\mathrm{D}$ trunk $) \mathrm{x}$ A trunk $\mathrm{x} 0.3+($ E lower limbs + I lower limbs + Ev lower limbs + D lower limbs) x A lower limbs x $0.4+$ Mucosal area score x Mucosal damage severity score. Total scores were between 0 and 93 points, according to the formula.

The patient's initial DASI score on admission was 9.9 points (Fig. 3). The score prior to injection was 64 points (Fig. 3); meanwhile the rash area covered $80 \%$ of the body surface area. According to SCORNTEN scoring method (9), this case's score was 2 points and predicted mortality was $12.1 \%$. Following the first injection of etanercept, the fever was under control within $1 \mathrm{~h}$. The rash was under control within $24 \mathrm{~h}$ and no new rashes appeared. The score was 59.4 points (Fig. 3). The rash further

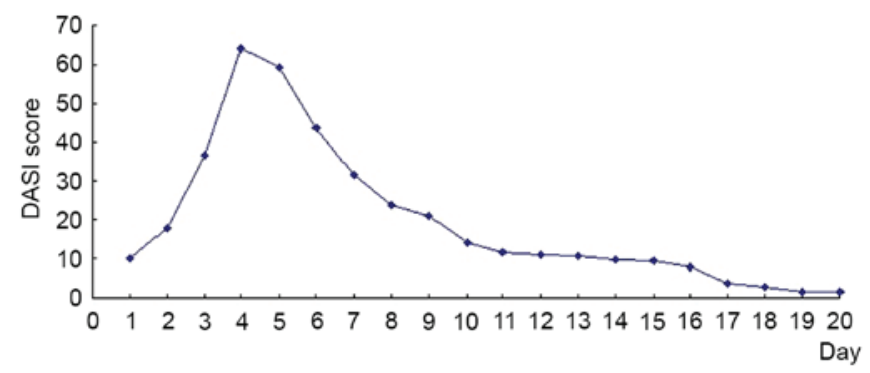

Figure 3. Patient's drug eruption area and severity index changes following hospital admittance.
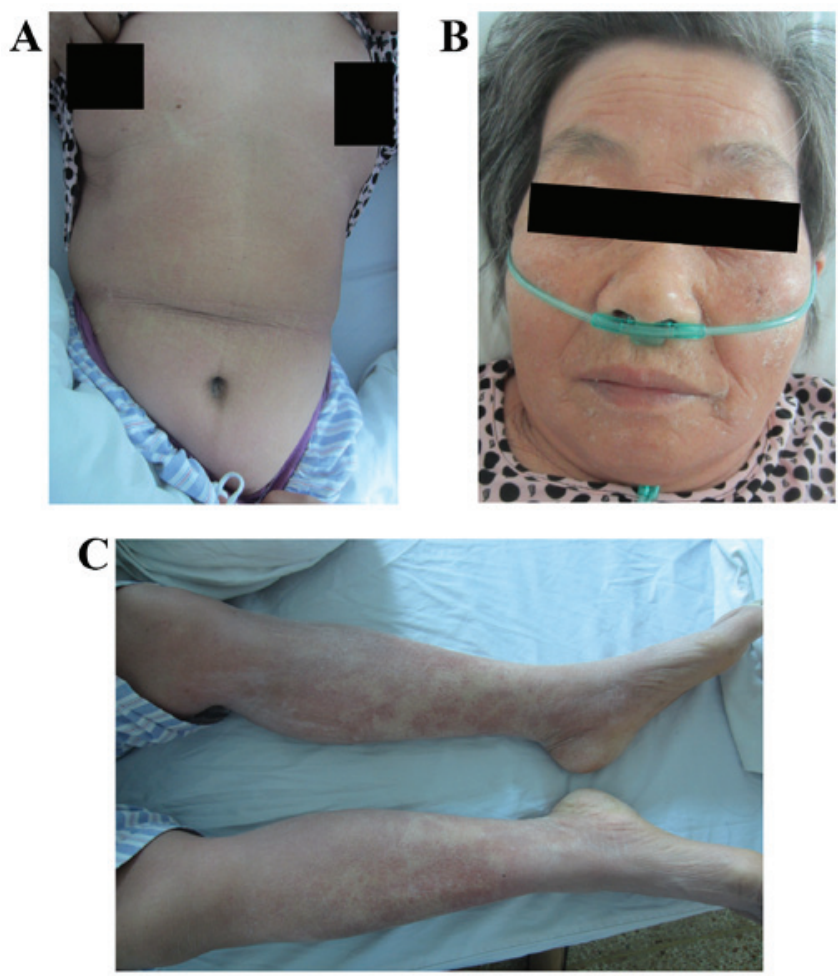

Figure 4 . At $72 \mathrm{~h}$ after injection, the rash significantly improved, became dark red and was not as itchy as before. Conjunctival secretion reduced and slight desquamation appeared on the face. The patient's (A) torso, (B) face and $(\mathrm{C})$ legs are presented to demonstrate the extent of the rash.

improved over the next $72 \mathrm{~h}$. The rash color turned dark and the itchiness and conjunctival secretion markedly reduced. Slight scaling appeared on the face and some normal skin was revealed. The rash covered $45 \%$ of the whole body surface area (Fig. 4A-C) and scored 23.9 points (Fig. 3). The patient did not feel itchy and clear desquamation began to appear on the head, face and trunk following $96 \mathrm{~h}$. Conjunctival erosion was almost recovered and the score was 20.9 points (Fig. 3). Two herpes simplex sores appeared separately on the right lip and the back of the right shoulder-back on day 5 following the injection. The patient was treated with penciclovir cream, and recovered 1 week later. The rash on the patient's limbs, in particular the lower limbs, faded markedly by day 7 following injection and the target-like erythema disappeared. The trunk and limbs markedly began to scale, the face and neck returned to normal and the score was 10.9 points (Fig. 3). The majority of the patient's skin returned to normal and the primary rash was shedding on 
the lower limbs on day 14 (Fig. 5A-C), and the score was 1.7 points (Fig. 3). On day 16, the patient was discharged and the score was 0.4 points (Fig. 3). Following admission, the water and electrolyte balance was maintained at 2,000-2,500 ml daily fluid infusion to allow adequate urine output. The patient was also instructed drink milk and was treated with an intravenous drip of compound glycyrrhizin ( $80 \mathrm{ml}$ daily). The patient was asked to gargle with chlorhexidine solution and protect her eyes with chlortetracycline eye ointment. During the treatment, the patient presented with no nausea, vomiting, diarrhea, hematochezia, cough or abdominal pain.

Laboratory examination on the first day of admission indicated that the leukocyte count was $5.03 \times 10^{9}$ cells/l (normal, 4.00-10.0x $10^{9}$ cells/1; Fig. 6) and the percentage of eosinophils was 5.2\% (normal, 0.5-5.0\%; Fig. 6). Prior to etanercept injection, the leukocyte count rose to $24.70 \times 10^{9} / 1$ (Fig. 6) and the percentage of neutrophils increased to $93.0 \%$. The percentage of eosinophils was 1.9\% (Fig. 6) and biochemical indicators were normal, with the exception of lactate dehydrogenase (LDH) being slightly increased at $369 \mathrm{U} / 1$ (normal, 109-245 U/1), and total protein slightly dropped to $56.7 \mathrm{~g} / \mathrm{l}$ (normal, 60.0-85.0 g/l).

The peripheral blood eosinophil count continued to rise and the percentage peaked at $23.6 \%$ (Fig. 6) following the third injection of etanercept. When the patient was discharged, the percentage of eosinophils remained higher than normal and reached up to $12.4 \%$ (Fig. 6). The patient's hepatic and renal function remained normal during hospitalization. On day 3 following the injection of etanercept, LDH was slightly increased at $271 \mathrm{U} / 1$ (normal, 109-245 U/1), serum total protein was lowest at $49.6 \mathrm{~g} / \mathrm{l}$ (normal, 60.0-85.0 g/l) and albumin was lowest at $30.6 \mathrm{~g} / \mathrm{l}$ (normal, $35.0-55.0 \mathrm{~g} / \mathrm{l}$ ).

Albumin was not administered but the patient was instructed to drink more milk. The level of albumin returned to normal when reviewed. On day 9 following injection, the level of triglycerides rose to $2.35 \mathrm{mmol} / 1$ (normal, $0.33-1.70 \mathrm{mmol} / \mathrm{l}$ ) and returned to normal when the patient was discharged. Written informed consent was obtained from the patient for publication of the images and medical data in this report.

\section{Discussion}

The patient in the current study presented with generalized edematous erythema, lymphadenectasis, fever, severe itch, conjunctival erosion and increased levels of leukocytes and eosinophils in the peripheral blood following antibiotic treatment. Parts of the rash were target-like and therefore, the diagnosis was severe erythema multiforme-type drug eruption (Stevens-Johnson syndrome, SJS). According to the probability of drug eruption, amoxicillin allergy was the most probable cause.

Severe erythema multiforme-type drug eruption is a serious adverse drug reaction and it has a risk of mortality. According to previous studies $(10,11)$, the average mortality rate for SJS is $1-5 \%$, and the risk of mortality is higher in elderly patients. The most common drugs that cause allergic reactions are penicillin, sulfonamides and nonsteroidal anti-inflammatory drugs $(12,13)$. TNF- $\alpha$ is a cytokine secreted by macrophages, lymphocytes and other immune cells that are activated by endotoxins. TNF- $\alpha$ is the strongest anti-tumor
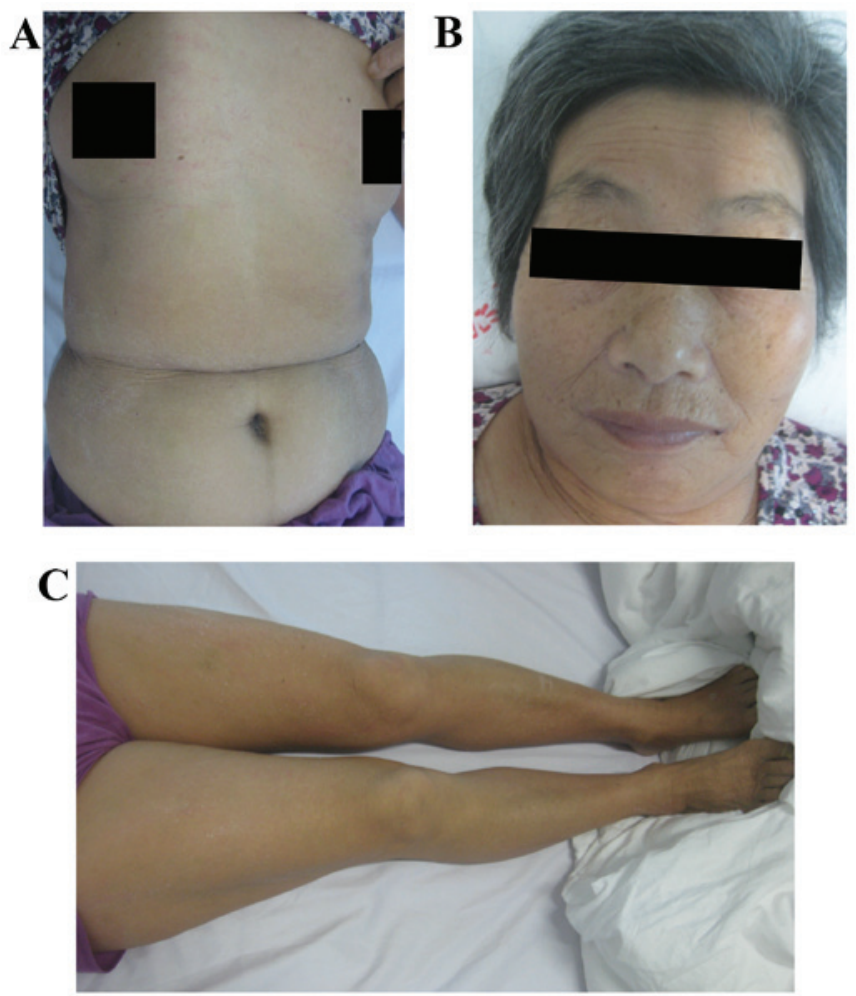

Figure 5. On day 14 after injection, the majority of the patients skin had returned to normal. The patient's (A) torso, (B) face and (C) legs are presented to demonstrate the extent of the rash.

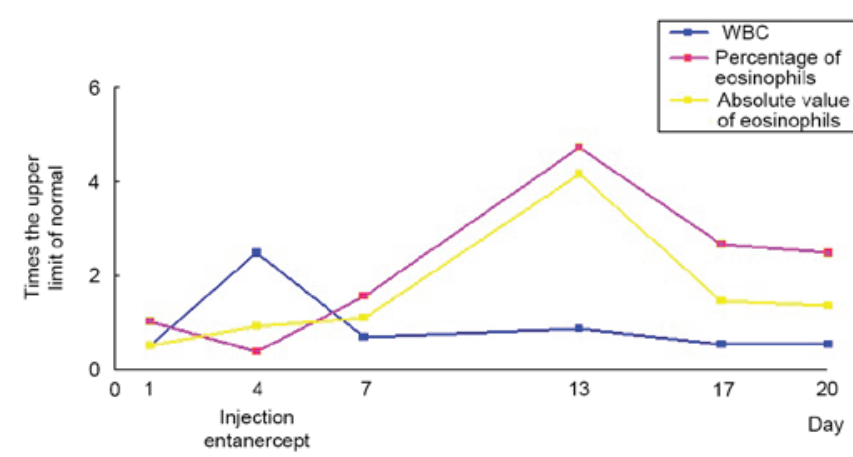

Figure 6. Changes in the level of white blood cells and eosinophils during hospitalization.

and anti-inflammation cytokine identified, thus far. TNF- $\alpha$ has a role in the occurrence and development of a number of different diseases and illnesses, including cancer, infection, fever, endotoxin shock, autoimmune diseases and transplant rejection (14-18). The more severe the trauma is, the higher the level of TNF- $\alpha$ is $(19,20)$.

Epidermal necrosis in SJS and TEN is considered to be caused by cytotoxic T cells. Perforin, which is released with TNF- $\alpha$ and other cytokines, may induce keratinocyte necrosis. Paquet et al (21) demonstrated that there is a high expression of TNF- $\alpha$ in the rashes of patients with TEN and hypothesized that TNF- $\alpha$ mediates apoptosis. Carr et al (22) revealed that TNF- $\alpha$ was detected in the rashes of exanthematous drug eruption of patients with HIV infection and the positive rate was $\sim 45 \%$. Nassif et al (23) indicated marked TNF- $\alpha$ expression in 
the blister fluid of patients with TEN. Paul et al (24) observed that the rashes of 5 patients with TEN and SJS-TEN overlap and demonstrated that all the rashes exhibited full-thickness necrosis of epidermal keratinocytes. This was hypothesized to be due to extensive apoptosis following the release of TNF- $\alpha$, which may enhance this toxicity. This suggests that the inhibition of apoptosis may be an important means of treating TEN.

TNF- $\alpha$ is a pro-inflammatory cytokine. The pathogenesis of TNF- $\alpha$ may involve severe eruption due to the following aspects: i) As the drug is absorbed into the body, it activates drug-specific T cells that secrete TNF- $\alpha$, stimulate keratinocytes and endothelial cells and mediate the immune responses of skin and mucosa; ii) TNF- $\alpha$ mediates type IVa hypersensitivity; and iii) in TEN, TNF- $\alpha$ has a role in FasL-mediated keratinocyte apoptosis by activating inducible nitric oxide synthase (25).

TNF antagonists may block the biological activity of TNF by specific binding, which may function to control the inflammation, leading to recovery from the disease. Previously, there have been a number of cases of single use TNF factor antagonists to successfully treat severe drug eruption, globally. Hunger et al (2) used single infliximab $(5 \mathrm{mg} / \mathrm{kg})$ to treat a 69 -year-old female patient with TEN, and successfully identified the point when the recovery time was significantly shorter than the gamma globulin. Kreft et al (7) used infliximab to successfully treat a case of celecoxib-induced TEN. Chen et al (8) injected etanercept every other day for 16 days and successfully treated a patient with Dress accompanied diabetes.

In the present study, the patient recovered and was discharged on day 16, following subcutaneous injections of $25 \mathrm{mg}$ (initial dose, $50 \mathrm{mg}$ ) etanercept every 3 days for 6 times. The total amount of etanercept treatment included seven injections. It is evident that TNF- $\alpha$ antagonist is able to target and inhibit the early inflammatory mediator TNF- $\alpha$ in a timely and effective manner, terminate the inflammatory cascade and then block the pathogenesis of drug eruption. Therefore, TNF- $\alpha$ antagonist is effective in the treatment of severe drug eruption.

Prior to treatment by injection, the total level of leucocytes and neutrophils markedly increased. Considering that the patient presented with no clear evidence of infection, it was speculated that this was associated with increased interleukin (IL)-8 in the drug eruption pathogenesis. IL-8 has the effect of stimulating neutrophil chemotaxis and releasing lysosomes. Therefore, no antibiotics were used during treatment. TNF- $\alpha$ is able to induce IL-8. In this case, the patient's leukocytes and neutrophils quickly returned to normal following treatment with the TNF- $\alpha$ antagonist. In the follow-up examinations, the level of leukocytes and neutrophils remained within the normal range. This may be because the TNF- $\alpha$ antagonist inhibits TNF- $\alpha$, reduce IL- 8 and then inhibit the chemotaxis of neutrophils.

The peripheral blood eosinophil continued to rise following the injection of TNF- $\alpha$ antagonist in the present case and reached its peak on day 9 following the injection. From then, it declined slowly, but remained significantly higher than normal when the patient was discharged (Fig. 6). This observation is the same as Chen et al 's (8). This suggests that TNF antagonist is a safe, fast and effective therapeutic for severe drug eruption, but it may not prevent the rise of peripheral blood eosinophils, as they are not affected by the treatment.
In addition, in the process of using TNF- $\alpha$ antagonist, the patient's gastrointestinal symptoms were continuously improved and melena did not recur. It indicated that TNF- $\alpha$ antagonist does not affect the prognosis of underlying diseases such as upper gastrointestinal bleeding and peptic-ulcer in the present case.

In gastroenterology, anti-HP treatment typically uses penicillin antibiotics. Penicillin is associated with a higher probability of leading to drug allergies or even severe drug eruption (26,27). As the patients using anti-HP therapy typically also have upper gastrointestinal bleeding, the contraindication is to use glucocorticoids. It is therefore, difficult to treat drug eruption in these cases. The patient of the current study was treated with a TNF- $\alpha$ antagonist, which achieved promising results.

In conclusion, the present case of drug eruption was healed within 16 days of injection treatments and this treatment did not affect the underlying diseases, including gastrointestinal ulcer bleeding. Therefore, TNF- $\alpha$ antagonist may be a good treatment in similar situations. TNF- $\alpha$ antagonist opens new perspectives for the treatment of severe drug eruption. Due to our treatment being administered to a single case, well-conducted studies of the efficacy and safety of such treatment are urgently needed.

\section{References}

1. Kardaun SH and Jonkman MF: Dexamethasone pulse therapy for Stevens-Johnson syndrome/toxic epidermal necrolysis. Acta Derm Venereol 87: 144-148, 2007.

2. Hunger RE, Hunziker T, Buettiker U, Braathen LR and Yawalkar N: Rapid resolution of toxic epidermal necrolysis with anti-TNF-alpha treatment. J Allergy Clin Immunol 116: 923-924, 2005.

3. Engelhardt SL, Schurr MJ and Helgerson RB: Toxic epidermal necrolysis: An analysis of referral patterns and steroid usage. J Burn Care Rehabil 18: 520-524, 1997.

4. Guibal F, Bastuji-Garin S, Chosidow O, Saiag P, Revuz J and Roujeau JC: Characteristics of toxic epidermal necrolysis in patients undergoing long-term glucocorticoid therapy. Arch Dermatol 131: 669-672, 1995.

5. Schneck J, Fagot JP, Sekula P, Sassolas B, Roujeau JC and Mockenhaupt M: Effects of treatments on the mortality of Stevens-Johnson syndrome and toxic epidermal necrolysis: A retrospective study on patients included in the prospective EuroSCAR study. J Am Acad Dermatol 58: 33-40, 2008.

6. Moghadam-Kia S and Werth VP: Prevention and treatment of systemic glucocorticoid side effects. Int J Dermatol 49: 239-248, 2010.

7. Kreft B, Wohlrab J, Bramsiepe I, Eismann R, Winkler M and Marsch WC: Etoricoxib-induced toxic epidermal necrolysis: Successful treatment with infliximab. J Dermatol 37: 904-906, 2010.

8. Chen LL, Lu DD, Shi X, Sun XD, Xie LX, Chen XJ, Ding L, Jin WJ and Wang YF: Drug rash with eosinophilia and systemic symptoms controlled by tumor necrosis factor- $\alpha$ antagonist: A clinical observation. Zhonghua Pi Fu Ke Za Zhi 46: 621-625, 2013 (In Chinese).

9. Bastuji-Garin S, Fouchard N, Bertocchi M, Roujeau JC, Revuz J and Wolkenstein P: SCORTEN: A severity-of-illness score for toxic epidermal necrolysis. J Invest Dermatol 115: 149-153, 2000

10. Harr T and French LE: Stevens-Johnson syndrome and toxic epidermal necrolysis. Chem Immunol Allergy 97: 149-166, 2012.

11. Miliszewski MA, Kirchhof MG, Sikora S, Papp A and Dutz JP: Stevens-Johnson syndrome and toxic epidermal necrolysis: An analysis of triggers and implications for improving prevention. Am J Med 129: 1221-1225, 2016.

12. Lalosevic J, Nikolic M, Gajic-Veljic M, Skiljevic D and Medenica L: Stevens-Johnson syndrome and toxic epidermal necrolysis: A 20-year single-center experience. Int J Dermatol 54: 978-984, 2015.

13. Greenberger PA: Drug allergy. J Allergy Clin Immunol 117 (2 Suppl Mini-Primer): S464-S470, 2006. 
14. Waters JP, Pober JS and Bradley JR: Tumour necrosis factor in infectious disease. J Pathol 230: 132-147, 2013.

15. Stefferl A, Hopkins SJ, Rothwell NJ and Luheshi GN: The role of TNF-alpha in fever: Opposing actions of human and murine TNF-alpha and interactions with IL-beta in the rat. Br J Pharmacol 118: 1919-1924, 1996.

16. Nakayama M, Niki Y, Kawasaki T, Takeda Y, Horiuchi K, Sasaki A, Okada Y, Umezawa K, Ikegami H, Toyama Y and Miyamoto T: Enhanced susceptibility to lipopolysaccharide-induced arthritis and endotoxin shock in interleukin-32 alpha transgenic mice through induction of tumor necrosis factor alpha. Arthritis Res Ther 14: R120, 2012.

17. Doss GP, Agoramoorthy G and Chakraborty C: TNF/TNFR: Drug target for autoimmune diseases and immune-mediated inflammatory diseases. Front Biosci (Landmark Ed) 19 1028-1040, 2014

18. Azzawi M, Hasleton PS and Hutchinson IV: TNF-alpha in acute cardiac transplant rejection. Cytokines Cell Mol Ther 5: 41-49, 1999.

19. Esposito $\mathrm{E}$ and Cuzzocrea S: TNF-alpha as a therapeutic target in inflammatory diseases, ischemia-reperfusion injury and trauma. Curr Med Chem 16: 3152-3167, 2009.

20. He M, Dong LM and Hou XB: Time-related expression of IL-6, IL-8 and TNF-alpha following explosive injury to rabbit's chest. Fa Yi Xue Za Zhi 30: 85-87, 2014 (In Chinese).

21. Paquet P, Nikkels A, Arrese JE, Vanderkelen A and Piérard GE: Macrophages and tumor necrosis factor alpha in toxic epidermal necrolysis. Arch Dermatol 130: 605-608, 1994.
22. Carr A, Vasak E, Munro V, Penny R and Cooper DA: Immunohistological assessment of cutaneous drug hypersensitivity in patients with HIV infection. Clin Exp Immunol 97: 260-265, 1994.

23. Nassif A, Bensussan A, Dorothée G, Mami-Chouaib F, Bachot N, Bagot M, Boumsell L and Roujeau JC: Drug specific cytotoxic T-cells in the skin lesions of a patient with toxic epidermal necrolysis. J Invest Dermatol 118: 728-733, 2002.

24. Paul C, Wolkenstein P, Adle H, Wechsler J, Garchon HJ, Revuz J and Roujeau JC: Apoptosis as a mechanism of keratinocyte death in toxic epidermal necrolysis. Br J Dermatol 134: 710-714, 1996.

25. Viard-Leveugle I, Gaide O, Jankovic D, Feldmeyer L, Kerl K, Pickard C, Roques S, Friedmann PS, Contassot E and French LE: TNF- $\alpha$ and IFN- $\gamma$ are potential inducers of Fas-mediated keratinocyte apoptosis through activation of inducible nitric oxide synthase in toxic epidermal necrolysis. J Invest Dermatol 133: 489-498, 2013.

26. Lin YF, Yang CH, Sindy H, Lin JY, Rosaline Hui CY, Tsai YC, Wu TS, Huang CT, Kao KC, Hu HC, et al: Severe cutaneous adverse reactions related to systemic antibiotics. Clin Infect Dis 58: 1377-1385, 2014

27. Strom BL, Carson JL, Halpern AC, Schinnar R, Snyder ES, Shaw M, Tilson HH, Joseph M, Dai WS, Chen D, et al: A population-based study of Stevens-Johnson syndrome. Incidence and antecedent drug exposures. Arch Dermatol 127: 831-838, 1991. 